\title{
Some Physical Chemical Properties of Aqueous Solutions of Soaps and Soapless Detergents
}

\author{
By Gopal S. Hattiangdi, ${ }^{1}$ William W. Walton, and James I. Hoffman
}

\begin{abstract}
A study has been made of the effect of concentration on the surface tension, electrical conductance, $\mathrm{pH}$, opacity, and foam formation of aqueous solutions of 30 typical commercial soaps and 6 popular soapless detergents. The soaps included the following types: Toilet, "medicated," glycerin, coco, washing (laundry), and shaving soaps. The results are explained on the basis of the colloidal nature of the solutions and the soap phases that have been found in the solid soaps by studies of X-ray diffraction and electron microscopy. The significance of the results in terms of micelle formation and the relationship between micelles and detergent action are discussed.
\end{abstract}

\section{Introduction}

Running water was the earliest "detergent" used, the cleansing being caused mainly by mechanical action and partly by solvent action. The relatively superior washing action of aqueous solutions, suspensions, and pastes of various herbs, plants, and nuts was quickly realized, to be followed a few centuries thereafter by the manufacture and use of saponified oils and fats (soap). In more recent times, synthetic soapless detergents are being commonly used by the housewife for laundering, dish-washing, and cleansing in general. Whether it is the ancient herb, the more recent soap, or the modern soapless synthetic compound, the cleansing action in all cases is based upon the same principle - the replacement of the undesired dirt by a detergent, leaving the dirt in a suspended and protected state so that it is easily removed by running water.

The criteria used in purchasing soaps and soapless washing compounds are usually their appearance and texture, the quantity of suds they produce, and, with some critical housewives, the action of the compound on the skin and hands. There are, however, no universally accepted physical-chemical standards to which soaps are referred with regard to their washing power. Some

1 Guest worker, Section of Surface Chemistry. pertinent experimental methods suggest themselves if the prerequisites for efficient washing are critically reviewed and analyzed.

In trying to wash a greasy dish or a dirty fabric, a superior wetting or surface-active agent is needed. After the greasy dirt is removed from the surface of the dish or fabric, the dirt particles must be suspended in the aqueous medium. This can be achieved by compensating the charge around the particles. When the charge is neutralized, both the uncharged dirt particles and the clean surface of the dish or fabric must be protected with an adsorbed film of the detergent so that the removal of the dirt with running water may be facilitated. The use of an efficient detergent also enhances the degree of solubilization of the insoluble dirt, owing to its sorption by, or inclusion inside, the colloidal particles of the detergent. The factual data on these factors may be obtained by making direct measurements of surface tension, interfacial tension, carbon number, gold number, and dye number.

Other factors, which may be considered significant by the housewife, are the quantity of suds produced (properties of foams), the irritation to the skin and hands (control of $\mathrm{pH}$ ), and the clarity of the solution (opacity of turbidity).

The literature is profuse with physical-chemical data for aqueous solutions of pure alkali soaps, stress being laid in most of these investigations on 
the colloid chemical viewpoint. Krafft and Stern $[4]^{2}$ suggest that soaps must be in solution in order to exhibit appreciable detergent action. McBain [6] has shown conclusively that the detergent action of aqueous soap solutions is due to their colloidal nature, and that this action is influenced by the chemical constitution of the detergent only insofar as this affects their behavior as colloids. Investigations conducted during the past 10 years have shown, however, that sodium soaps of the saturated fatty acids display probably three alternative crystal forms, as well as a sequence of about 5 or 10 stable mesomorphic forms, each having different and unique physical-chemical properties [9].

The purpose of this paper is to present data for several physical-chemical properties of aqueous solutions of numerous commercial soaps and soapless detergent materials, and to correlate these with the structure and phase nature of the solid soaps as revealed by investigations with X-ray diffraction [2] and electron microscopy [3], and to interpret the significance of the results in terms of fundamental colloid chemical hypotheses.

\section{Materials Used}

Thirty typical commercial soaps (toilet, "medicated," glycerin, coco, washing (laundry), and shaving soaps) and six popular synthetic soapless detergents were investigated. The commercial soaps were the same as those used in previous investigations $[2,3]$. Of the soapless detergents, two (D1 and D5) are reported by their manufacturers to be nonalkaline compounds, one (D6) as containing "a special ingredient that dissolves grease on contact," and one (D2) as a new kind of soap. The active principles of the soapless detergents D1, D3, D4, and D5 are probably a fatty alcohol sulfate, an alkyl-aryl sulfonate plus an alcohol sulfate, a sulfated monoglyceride of coconut oil, and an alkyl-aryl sulfonate, respectively.

\section{Experimental Techniques}

Aqueous solutions corresponding to 10, 5, 2.5, $1.25,0.625,0.313,0.156$, and 0.078 percent, by weight, of the soap or soapless detergent were prepared by dissolving the appropriate amount

${ }^{2}$ Figures in brackets indicate the literature references at the end of this paper. of the soap (or soapless detergent) in hot $\left(80^{\circ}\right.$ to $\left.100^{\circ} \mathrm{C}\right)$ distilled water. The solution was then cooled, transferred to a $250-\mathrm{ml}$ volumetric flask, and fresh cold distilled water added until the solution reached the standard mark.

\section{Surface Tension}

A du Nouy tensiometer was used for making surface-tension measurements. The platinum ring had a circumference of $6.014 \mathrm{~cm}$ and a ratio of $R / r$ equal to 53.5. The instrument was calibrated against known standard weights and also against conductivity water.

\section{Electrical Conductance}

The electrical conductance was measured with a dip-type conductivity cell, with a cell constant of 0.8307 , and a conductivity bridge manufactured by the Solar Manufacturing Corporation, New York (model-RC1; 20 watts; 100 to 125 volts; 50 to 60 cycles). The null point was detected with the help of a magic-eye, which flickered when the switch multiplier was turned from one position to another, indicating that the null point occurred in that region. The multiplier was then kept steady and the resistance dial moved until the green portion of the magic-eye receded to a maximum. This was the null point. In the absence of accurate knowledge of the exact equivalent weights of the commercial soaps and soapless detergents, values for the specific conductance rather than the equivalent conductivity have been calculated and are presented in this paper. However, the data in figure 2 were calculated by the equation

$$
\text { weight conductance }=\frac{1,000 \times K}{C},
$$

where $K$ equals specific conductance, and $C$ equals weight of soap or soapless detergent in grams per liter. This was done to obtain curves similar to those that would be obtained if equivalent conductance were plotted.

\section{3. $\mathrm{pH}$}

The $\mathrm{pH}$ of the various soap and soapless detergent solutions was measured with a glass-electrode $\mathrm{pH}$ meter. The instrument was standardized against standard solutions of Borax ( $\mathrm{pH}$ equals 9.18 at $25^{\circ} \mathrm{C}$ for a 0.01 -molar solution). 


\section{Opacity}

The opacity of the various solutions was measured with a Klett-Summerson photoelectric colorimeter. A projection type of electric lamp (100 watts; 110 volts) fitted with a green filter (540 millimicrons) was used as the source of light, and the current produced by the photocells was measured by means of a suspension wire type of galvanometer. The opacity of the system, that is the reciprocal of the intensity of transmitted light, was measured in terms of the divisions on the potentiometer slide-wire that are necessary to nullify the potential applied to the galvanometer.

\section{Foaming}

The rate of growth of foam was determined by measuring the height to which the foam rises under a standard bubbling rate. The bubbler was prepared as follows. A glass tube, 1/8 in. in diameter and $18 \mathrm{in}$. long, was sealed at one end with small pieces of copper wire $(0.01 \mathrm{~mm}$ diameter $)$ fused into it. When the end of the tube had cooled it was dipped into nitric acid, which dissolved the copper wire and left 20 fine holes of uniform diameter. The open end of the tube was then connected to an air-pressure valve via a flow regulator, and the bubbler was dipped into a $1,000-\mathrm{ml}$ measuring cylinder containing $200 \mathrm{ml}$ of the solution to be tested. The time was noted on a stop watch.

\section{Results and Discussion}

Data for aqueous solutions of some typical soaps and soapless detergents are presented in figures 1

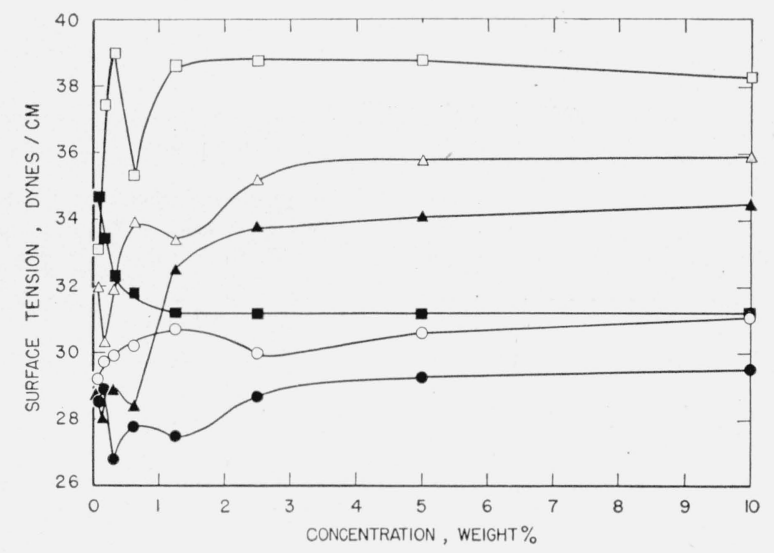

FIGURE 1. Variation of surface tension with concentration. T3 toilet soap; $\mathrm{C} 1$ coco soap; $\triangle \mathrm{G} 2$ glycerin soap; $\boldsymbol{\Delta}$ W 5 washing soap; $\square$ S5 shaving soap; $\square$ D5 soapless detergent.

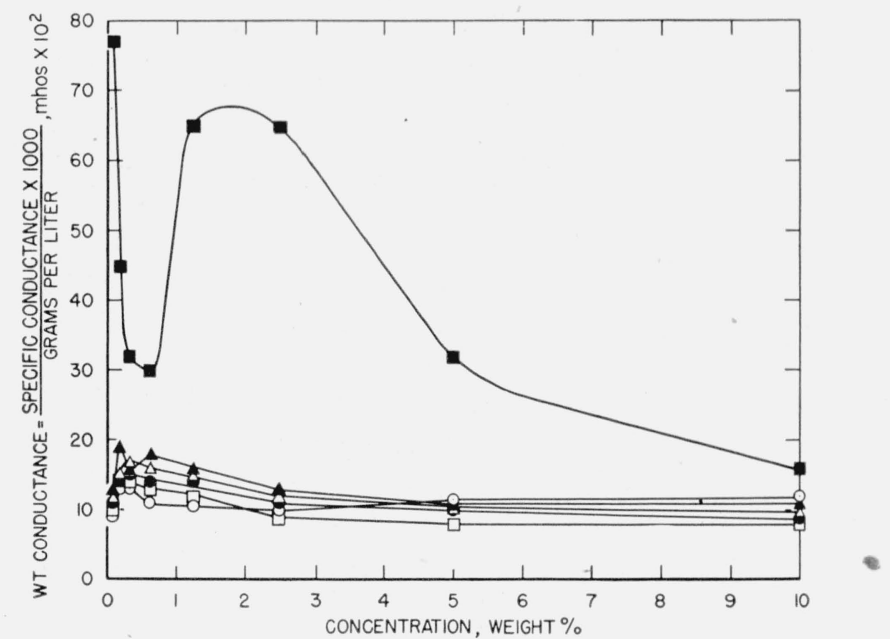

Figure 2. Variation of specific conductance with concentration.

T3 toilet soap; $\mathrm{C} 1$ coco soap; $\triangle \mathrm{G} 2$ glycerin soap; $\boldsymbol{\Delta}$ W5 washing soap; $\square$ S5 shaving soap; $\mathbf{D} 5$ soapless detergent.

to 4 , in which the surface tension, weight conductance, $\mathrm{pH}$, and opacity of the various solutions are plotted with respect to concentration. Data for all the soaps and soapless detergent solutions at a concentration of 2.5 percent are presented in tables 1 and 2, since such a tabulation permits quick comparison and evaluation.

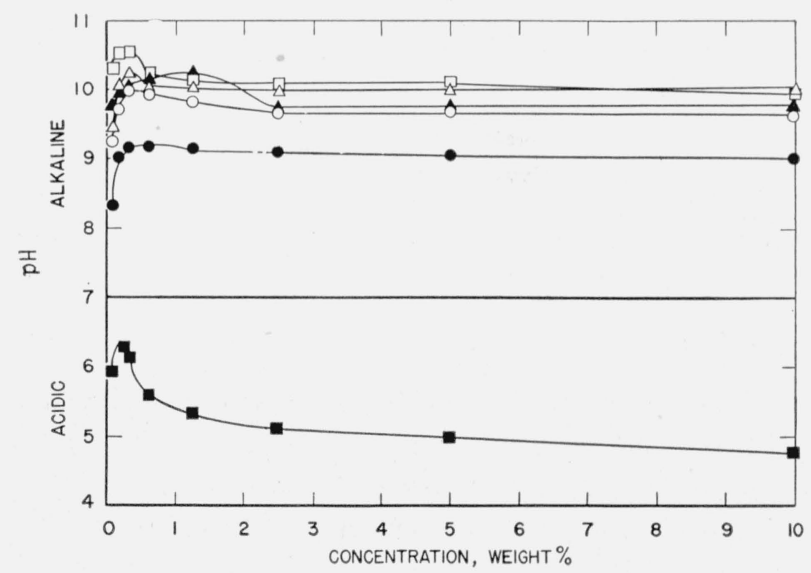

Figure 3. Variation of $\mathrm{pH}$ with concentration

$\bigcirc$ T3 toilet soap; $\bigcirc \mathrm{C} 1$ coco soap; $\triangle \mathrm{G} 2$ glycerin soap; $\mathbf{A}$ W5 washing soap; $\square$ S5 shaving soap; $\mathbf{D} 5$ soapless detergent.

\section{Surface Tension}

The curves obtained by plotting surface tension with respect to concentration are very similar for the various toilet soaps but differ somewhat from those for the coco, glycerin, washing, and shaving 
TABLE 1. Some physical-chemical properties of 2.5 percent aqueous soap solutions

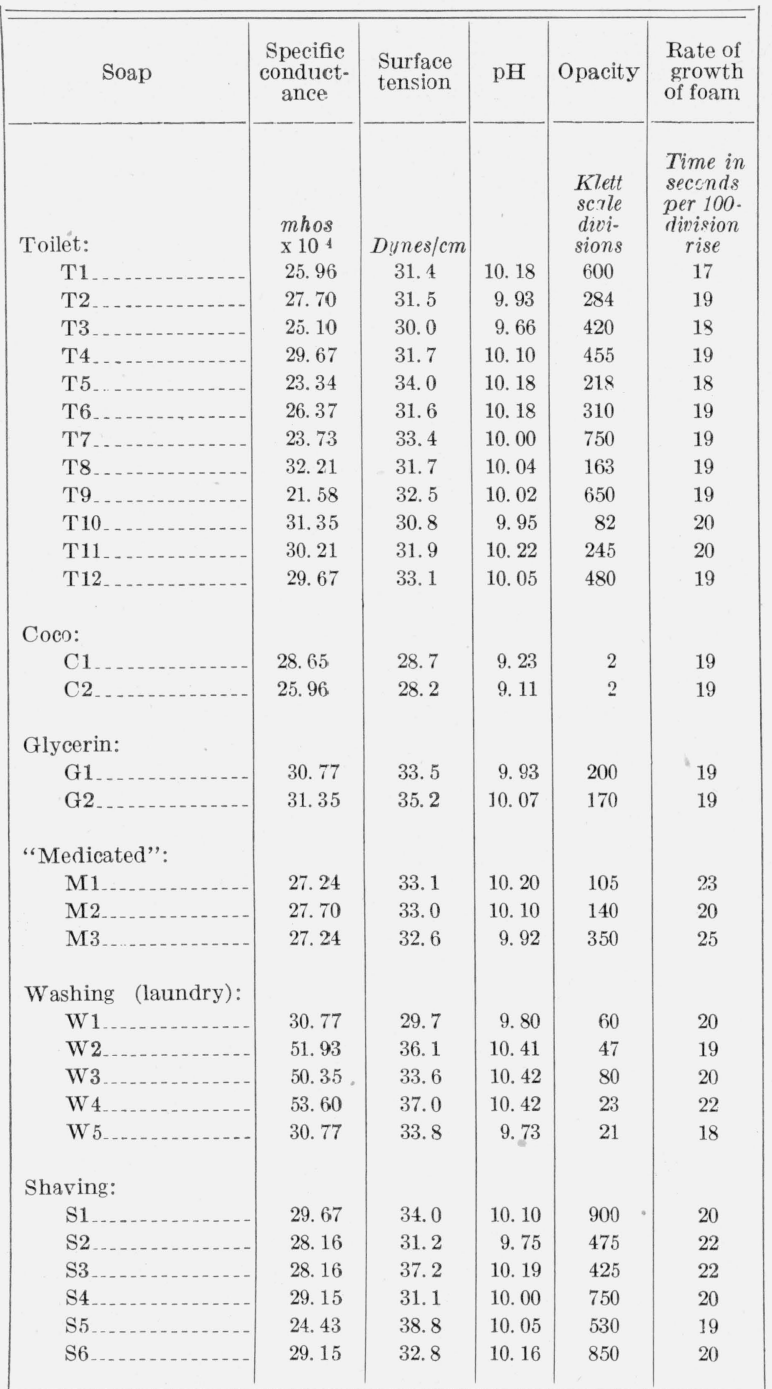

soaps. Solutions of the toilet soaps are characterized by a very low value of surface tension at small concentrations (about 0.1 to $0.2 \%$ ), which increases to a maximum value around 1.25 percent of soap content. The surface tension then decreases slowly to a minimum value, around 2.5 percent of soap, which either remains constant or increases very slowly as the soap content is increased. The curves thus show two minima, around 0.1 to 0.2 percent and 2.5 percent of soap.

The solutions of coco, glycerin, washing, and shaving soaps are characterized by the surface tension passing through a minimum value in very dilute solutions (about 0.2 to $0.3 \%$ of soap), and then following a somewhat irregular and indefinite path up to about 1.25 percent. The value of surface tension then increases somewhat to reach a maximum, which is either retained or decreases slightly as the concentration of the soap is increased. In contrast to the data for solutions of the toilet soaps, solutions of this group of soaps do not show a second minimum around 2.5-percent concentration.

In either case, the minima in the region of lower concentration are due to the surface film being saturated with a single layer of soap molecules, whereas the minima in the region of higher concentration indicate the formation of soap micelles with single or multiple charges. The steady value of surface tension obtained beyond a concentra-

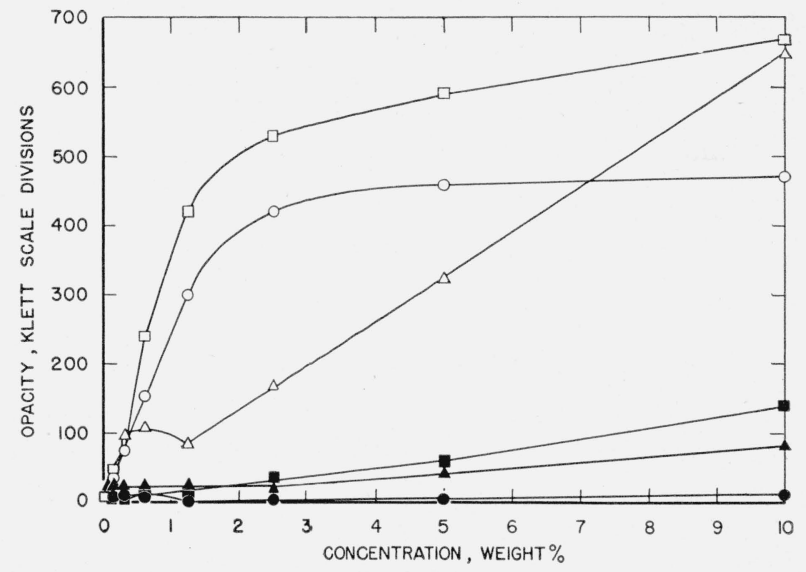

Figure 4. Variation of opacity with concentration.

T3 toilet soap; $\mathrm{C} 1$ coco soap; $\triangle$ G2 glycerin soap; $\Delta$ W5 washing soap; $\square$ S5 shaving soap; D5 soapless detergent.

TABLE 2. Some physical-chemical properties of 2.5-percent aqueous soapless detergent solutions

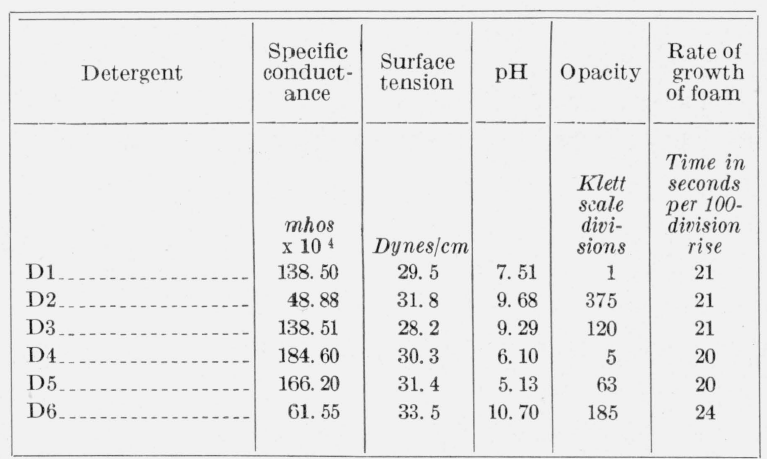


tion of about 2.5 to 4 percent of soap indicates that both the surface and the interior of the system are saturated with respect to the charged micelles. The exhibition of two regions of minimum surface tension for solutions of the toilet soaps and of only one such region for solutions of other types of soaps is probably to be explained as being caused by the presence of many sizes and kinds of colloidal particles in the former and of a constant particle size in the latter.

The solutions of the soapless detergents are characterized by a continuously decreasing value of surface tension with increasing concentration, an almost constant value being reached after about 1.25 percent. The shape and nature of the curves for the solutions of the soapless detergents are thus very different from tbose for the soap solutions. It seems reasonable to conclude from these data that the concentrations at which monolayers and ionic micelles are formed are much lower for the soapless detergent solutions tban those for the soaps. In terms of washing action, this may be interpreted as indicating that at lower concentrations the soapless detergents are better surface-active cleansing agents than the soaps.

\section{Electrical Conductance}

In the absence of data regarding exact equivalent weights of the soaps and soapless detergents, values of the specific conductance rather than of equivalent or molar conducitivity have been presented in tables 1 and 2 , and values of the weight conductance were plotted in figure 2 (see p. 363). The curves in figure 2 show that the conductance for the various soap solutions rises rather sharply in dilute solutions to a maximum around 0.25 percent and then decreases to about 2.5 percent, beyond which an almost constant value is obtained. The decrease in conductivity is due to the replacement of ions by colloidal particles which, although conducting, have a lower equivalent conductivity than the ions from which they are formed. The variations in the regions of maxima for the different soap solutions is probably caused by the presence of many sizes and kinds of colloidal particles in equilibrium with one another and to the shifting of the equilibrium position with concentration and soap type.

Whereas soap solutions exhibit a high electrical conductance in dilute solutions and low and de- creasing values with increasing concentration, solutions of the soapless detergents are characterized by very high values of conductance, which decrease rapidly up to 0.5 percent, then increase up to about 2 percent and finally decrease slowly with increasing concentration. The formation of colloidal particles thus appears to take place at very low concentrations in solutions of the soapless detergents, and the relatively high charge carried by them tends to make them better cleansing agents than commercial soaps in solutions of similar low concentrations.

\section{3. $\mathrm{pH}$}

The pH-concentration curves in figure 3 show that the values of $\mathrm{pH}$ for the soap solutions increase very sharply at low concentrations up to a maximum around 0.2 percent, followed by an equally sharp decrease and a minimum between 1 to 2.5 percent. A further increase in the concentration of the soap does not appreciably change the $\mathrm{pH}$ value.

Table 1 shows that the values of $\mathrm{pH}$ average about 10 for solutions of different soaps at a concentration of 2.5 percent. The presence of excess alkali in aqueous soap solutions favors the formation of ionic micelles [6] and helps to clarify the solutions by driving back hydrolysis and simultaneously increases the viscosity of the system. The relatively high alkalinity of the various soap solutions is caused by their readiness to hydrolyze in water and also by the free (unreacted) alkali in the commercial products. In either case, the alkali has an irritating effect upon the skin.

The solutions of the soapless detergents may be either acidic or alkaline, depending upon the nature of the major constituent of the products. As with the alkaline soap solutions, irritation to the skin may be caused by these alkaline or acidic solutions, and likewise solutions of these soapless detergents would seem to be best used in greater dilution.

\section{Opacity}

The opacity-concentration curves for the various soap-water systems are shown in figure 4 and indicate that the solutions at lower concentrations are clear and transparent. With increasing concentration the solutions become at first cloudy and translucent, and then dense and opaque at 
higher concentrations. It is presumptuous to attribute the turbidity of the soap solutions to their colloidal nature alone, because perfectly transparent solutions of various colloidal materials and translucent to opaque systems of crystalloidal substances are obtainable. Also, the insoluble acid soap may sediment out almost entirely on standing, leaving the bulk of the soap in a clear transparent solution, or it may remain partly or wholly suspended in particles whose dimensions range all the way from coarse suspensions down to the smallest resolvable particles.

In concentrated as well as dilute solutions, the opacity of the coco and glycerin soaps is substantially low. The former made from coconut oil, have a high laurate-myristate content; the opacity of solutions of soaps of the lower fatty acids is lower than that for corresponding solutions of soaps of the higher fatty acids. The high transparency of the glycerin soap solutions probably results from the presence of glycerin and sugars that are adsorbed on the surface of the soap particles and suitably change the index of refraction.

Solutions of the soapless detergents exhibit a very high transparency at both low and high concentrations. The initial low values of opacity occur in regions of concentration in which there is low surface tension and relatively high electrical conductance, and is probably due to the formation of monolayers and primary aggregates of the detergent molecules. The slight increase in opacity that starts around 1.25-percent concentration corresponds to the region in which the surface tension reaches a constant value, and the weight conductance levels off and then decreases. This is probably to be ascribed to the formation of secondary aggregates and ionic micelles.

\section{Foaming}

Measurements of the height to which the foam rises when a standard bubbling rate is employed indicate that there is no appreciable change in the rate of growth of foam with variations in the concentration of a given soap or soapless detergent. However, the rate is probably slightly slower in dilute solutions and somewhat faster in concentrated solutions.

The first signs of foam formation are shown in solutions containing approximately 0.0035 percent of soap, but at this extremely low concentration, the foam is very unstable and breaks down as soon as it is formed. Relatively stable foam is produced beyond about 0.007 percent. The formation of foam in these extremely dilute soap solutions shows that the stabilizing action of adsorption layers attains its maximum at surface concentrations much below the saturation of the surface layer (about 0.1 to $0.2 \%$ ). This stabilization arises not only by a mere enhancement of the mechanical properties of the surface layer, but also by a certain degree of mobility of the stabilizing molecules necessary for the rapid bridging of ruptures in the surface layers.

The foam produced from solutions containing 0.078 to 10 percent of soap or of soapless detergent is very stable and remains almost unchanged for an hour or more if the system is not disturbed by mechanical or thermal agitation. The requirements for a stable foam, enabling the bubbles to withstand mechanical shocks, are a lowering of the boundary tension by adsorption and suitable mechanical properties of the adsorbed stabilizing film. It is worth while remembering, however, that the power of foaming is not always or entirely to be ascribed to low surface tension. For instance, saponin solutions, whose surface tensions are only slightly less than that for water, bave a very great power of foaming on account of the formation of a solid or highly viscous film of an adsorbed material on the surface. It should be very worth while to examine the decay of foam under standard conditions of agitation and the stability of single bubbles liberated beneath the liquid surface, in the case of both the commercial soap and soapless detergent solutions.

\section{Correlation with Data on the Phase Nature and Structure of the Solid Soaps}

$\mathrm{X}$-ray diffraction studies of commercial soaps have shown [2] that the toilet and "medicated" soaps consist essentially of beta sodium palmitate and small amounts of omega sodium oleate, the coco soaps consist primarily of omega sodium laurate and sodium myristate, the shaving soaps consist essentially of two or more distinct phases (alpha, and/or beta, omega, and delta) of sodium palmitate, and the constituents of the glycerin and washing soaps are rather indefinite, the latter being probably in the omega phase. Electron microscopic investigations [3] also indicate that sodium palmitate may be in the washing soaps. 
With a view to obtaining some information regarding the general physical-chemical properties of the solid soaps and their phase nature, some qualitative experiments were conducted on the hardness of the soaps, their solubility, and their reaction to water. In general, it was found that:

The hardness of the soaps, based on the resistance offered to the fine edge of a steel spatula on application of a $100-\mathrm{g}$ load, decreases in the following order: Toilet, "medicated", washing, shaving.

The solubility of the coco, glycerin, and "medicated" soaps in warm water is the greatest of the groups and decreases in the order: coco, glycerin, medicated. The shaving soaps are more soluble than the toilet soaps and give transparent to translucent solutions on cooling, whereas the solutions of the toilet soaps set to opaque gels or curds. The solubility of all the five types of soaps is, however, relatively low in cold water and in hard water.

The percentage of soap rubbed off the cake (bar), when used in water against a laboratory towel, decreases in the following order: Shaving, toilet, washing.

On standing with water, the toilet and shaving soaps swell and disintegrate, whereas the washing soaps crack somewhat with little or no swelling.

Data in tables 1 and 2 show that the values of most physical-chemical properties are distinctly different for solutions of soaps as against those of the soapless detergents. Aside from this, the most striking behavior is perhaps the ability of the soapless detergents to dissolve rapidly and work in cold as well as in hard water. A considerable part of the soap used in household applications is probably employed to soften hard water in order to make the additional soap effective. On the contrary, the soapless detergents usually work equally efficiently in hard and in soft water, none being wasted in the task of softening the water.

\section{Micelles and Their Applications in Cleansing Action}

There is some difference of opinion as to the origin, manner of formation, structure, size, etc. of the colloidal aggregates termed loosely as "micelles". McBain [7] postulates at least two types of micelles (ionic and lamellar), whereas Hartley [1] postulates only one, namely, the ionic micelle. Solutions of different colloidal electro- lytes exhibit different phenomena with varying concentrations, and this behavior can be explained only if one assumes the existence of more than one type of micelle. It is the general belief that the ionic micelle in soap solutions, which is of significance in the present investigations, consists of a loose spherical aggregate of the paraffin chain ions with the polar groups oriented toward the aqueous phase. Recent investigations by Mattoon, Stearns, and Harkins [5] have shown that aqueous solutions of soaps and soapless detergents give a Bragg spacing that is independent of concentration and that is equal approximately to the double length of the molecule. They have designated this as the micelle thickness, their conception of a micelle being cylindrical, rather than spherical or lamellar.

The evidence for the existence of ionic micelles in solutions of commercial soaps and soapless detergents is based primarily on data on electrical conductance and surface tension. The former show the formation of micelles by the rapid decrease in conductivity with increasing concentration-the ionic micelles have a lower conductivity than the ions. Surface-tension data indicate the formation of monolayers of soap molecules on the surface of the solutions at very high dilutions and the formation of colloidal aggregates (ionic micelles) at slightly higher concentrations. Electrical conductance and surface-tension data for the solutions of the soapless detergents indicate that micelle formation reaches a very high value at relatively high dilutions.

The mechanism by which solutions of soaps and soapless detergents dissolve relatively insoluble substances, such as grease and dirt, can be explained simply in terms of either concept of the ionic micelle and the action of mixed monolayers: (a) the hydrocarbons in the dirt are dissolved in the interior of the micelle; (b) the polar compounds in the dirt are removed by adsorption on the surface of the micelle, with the hydrocarbon part inside the micelle and the polar groups in the aqueous phase; and (c) compounds that are soluble in water but insoluble in hydrocarbons are taken up by the polar group of the micelle and thus act as peptizing agents.

Since the degree of solubilization is related to the number and size of the micelles present [8], it seems reasonable to conclude that greater solubilization and hence more efficient cleansing may 
be achieved by using soaps in concentrations ranging from 2.5 to 4 percent and soapless detergents in less concentrated solutions (about $1.25 \%$ or less).

\section{References}

[1] G. S. Hartley, Trans. Faraday Soc. 31, 183 (1935); Colloidal electrolytes - a general discussion held by the Faraday Society, 1935; Aqueous solutions of paraffin-chain salts (Herman, Paris, 1936).

[2] G. S. Hattiangdi, J. Research NBS 42, 331 (1949 RP1972.

[3] G. S. Hattiangdi and M. Swerdlow, J. Research NBS 42, 343 (1949) RP1973.

[4] F. Krafft and A. Stern, Ber. deut. chem. Ges. 27, 1747 (1894); 32, 1584-(1899).
[5] R. W. Mattoon, R. S. Stearns, and W. D. Harkins, J. Chem. Phys. 16, 644 (1948).

[6] J. W. McBain, Third report on colloid chemistry and its general and industrial applications, British Association for the Advancement of Science, p. 2 to 31 (1920).

[7] J. W. NeBain, J. Phys. Chem. 43, 671 (1939); Nature 145, 702 (1940).

[8] J. W. McBain, Advances in colloid science 1, 99 (Interscience Publishers, Inc., New York, N. Y., 1942).

[9: R. D. Vold and M. J. Vold, Colloid chemistry 5, 266 (edited by J. Alexander, Reinhold Publishers Corporation, New York, N. Y., 1945).

Washington, January 18, 1949. 